\title{
Radiographic assessment in rheumatoid arthritis: From daily practice to clinical trials
}

\author{
Corina Mogosan 1,2, Luminita Enache ${ }^{1,2}$, Bianca Dumitrescu', Mihaela Agache ${ }^{1,2}$, \\ Catalina Ionescu ${ }^{1,2}$, Claudiu Popescu ${ }^{1,2}$, Catalin Codreanu ${ }^{1,2}$ \\ 1"Dr. Ion Stoia” Clinical Center of Rheumatic Diseases, Bucharest, Romania \\ 2"Carol Davila" University of Medicine and Pharmacy, Bucharest, Romania
}

\begin{abstract}
Imaging evaluation in rheumatoid arthritis (RA) has evolved significantly in last decades. Even if the modern imaging technics like ultrasound and magnetic resonance imaging have unequivocal place in the assessment of early disease (especially for synovial inflammation, bone oedema, erosions), radiographic evaluation of hands and feet is still the most widely used imaging technic for quantification of joint damage in RA.

In the current review we aim to address some practical issues related to the radiographic evaluation in RA from daily practice perspective, followed by a broader approach from the clinical trials point of view.
\end{abstract}

Keywords: rheumatoid arthritis, radiographic, erosion, joint space narrowing

\section{INTRODUCTION}

Is the presence of "erosions" the hallmark of rheumatoid arthritis (RA)? This issue has been addressed historically many times, as the knowledge about the formation of erosions in RA has evolved. It has been clearly stated by the experts that presence of erosions is not mandatory for the diagnosis of RA, as the disease can also evolve in a non-erosive state, especially at earlier stages.

Early diagnosis of RA can be often challenging, even if the cornerstone of the diagnosis is based on clinical assessment; the literature reports that the acute phase inflammatory markers (erythrocyte sedimentation rate (ESR) and $\mathrm{C}$ reactive protein (CRP) may be within normal range in up to $25 \%$ of the cases (1). The diagnosis of RA cannot fully rely on American College of Rheumatology / European League Against Rheumatism (ACR/EULAR) RA 2010 classification criteria, as they are not designed for diagnostic purposes (2). The role of modern imaging technics, like ultrasound (US) and magnetic resonance imaging (MRI) in detection of synovitis is to help the clinician to judge a case more accurately, behind the clinical evaluation only. Studies have outlined that patients initially assessed as having undifferentiated arthritis, based on clinical evaluation, have been classified as RA after US or MRI impacting on their disease management (3).

RA is characterized by proliferative symmetrical synovitis with consecutive joint damage, possibly associated with extra-articular manifestations. Classically, the articular involvement is a symmetrical polyarthritis of small joints, possibly also affecting large joints. Joint damage is characterised by synovial thickening, bone oedema, bone erosions, joint space narrowing, joint subluxations and bony ankylosis; as disease progresses, specific deformities are clinically visible. The small joints of the hand and feet are commonly affected in early disease (5). The presence of peripheral joint erosions is associated with cervical spine involvement (4). Cranial settling and atlanto-axial subluxations pose high risk for cervical cord compression (6).

Joint destruction develop early in RA evolution, is progressive and do not correlate with symptoms and serological inflammation (7). Damage seen on radiographs has been shown to be an important predictor of 


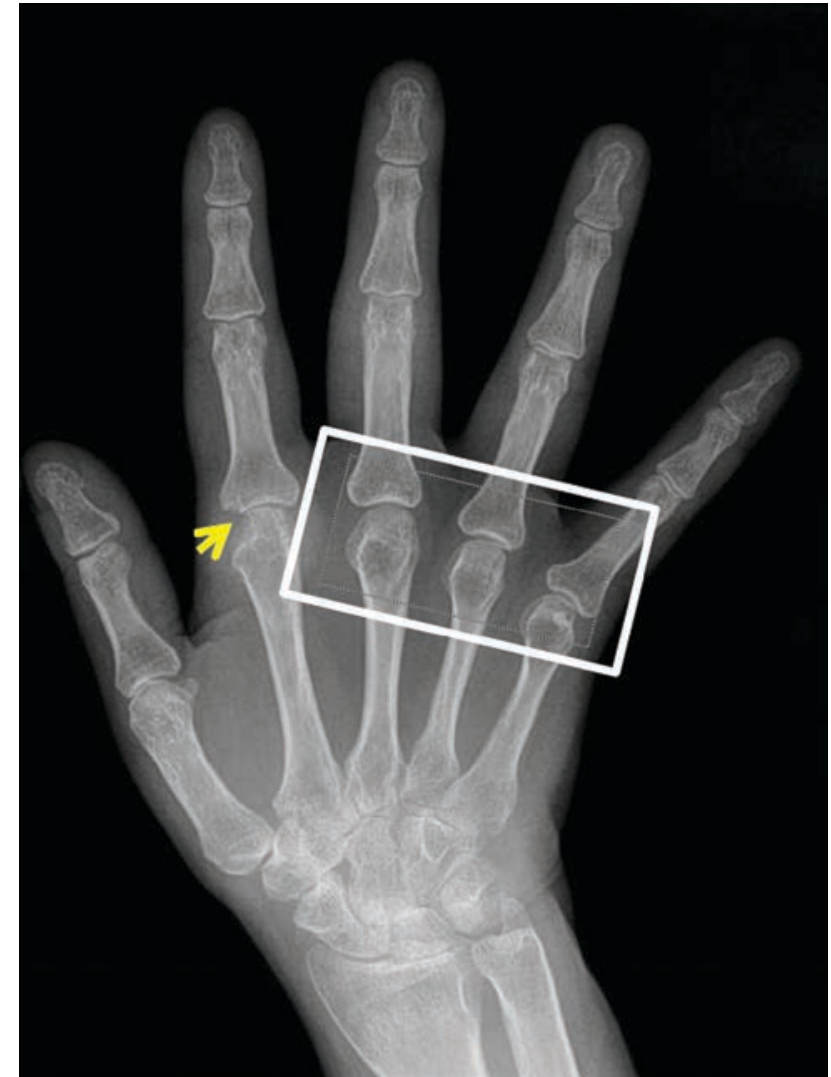

FIGURE 1. Swelling of the soft tissue (MCP 2,3), periarticular osteoporosis white (box), small erosion MCP2 (yellow arrow) in a 30yrs RA female patient, after one year of disease onset (image from the collection of Clinical Center of Rheumatic Diseases "Dr. Ion Stoia”)

clinical outcome and future progression (8). Consequently, after RA diagnosis the main treatment goal is achieving disease remission or a low disease activity state in order to prevent or to limit joint destruction.

Conventional radiology has been used for long time in the evaluation of damage in inflammatory arthritidis and should be used as the primary tool owing to its large accessibility and cost effectiveness. It has many advantages compared with other imaging modalities, such as the ability to image multiple joints simultaneously with minimum radiation exposure (9). Usually, the presence of erosions on hands and feet $\mathrm{x}$-rays are as late as one to two years after disease onset (10). However, often there is a delay in the patient's first visit to the rheumatologist from the time of symptom onset, which could range from several months to more than a year (11), so there is a possibility that radiographs changes can be detected at diagnosis.

\section{RADIOGRAPHICAL FEATURES}

Considering the disease pathology and evolution, the corresponding radiographical features can be described:

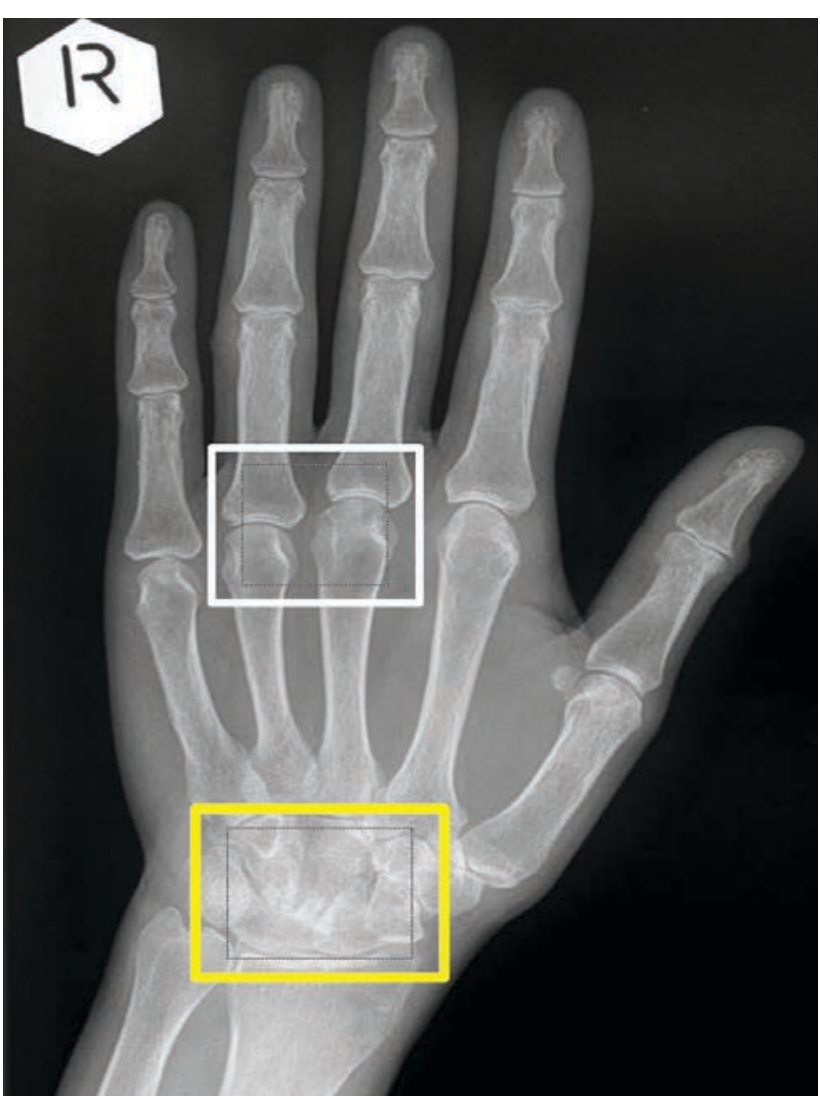

FIGURE 2. Uniform joint space narrowing of the $M C P 3,4$ (white box), severe joint space narrowing with bony ankylosis of the carpal bones (yellow box) in a 47 female RA patient at 2 years after disease onset (image from the collection of Clinical Center of Rheumatic Diseases "Dr. Ion Stoia”)

- swelling of the soft tissue is the x-ray correspondent of the clinical arthritis (Figure 1);

- periarticular osteoporosis (decrease in radiographic density in the osseous structure surrounding the affected joints) is a very early imaging sign of RA and is determined by the joint inflammation (with local release of inflammatory cytokines) (12) (Figure 1);

- uniform joint space narrowing, from mild to severe, progressing to bony ankylosis, is related to the damage of the hyaline cartilage, with thinning and ulceration (Figure 2);

- bone erosions are the imaging hallmark of the RA; they are typically located at the interface between pannus and bone at the joint margins and subchondral bone; erosions have different sizes, depending on disease duration and individual factors, from small to large, compromising the structure of articular bone components (Figure 3); their development is related to the persistent proliferative synovitis and activation of osteoclasts; 


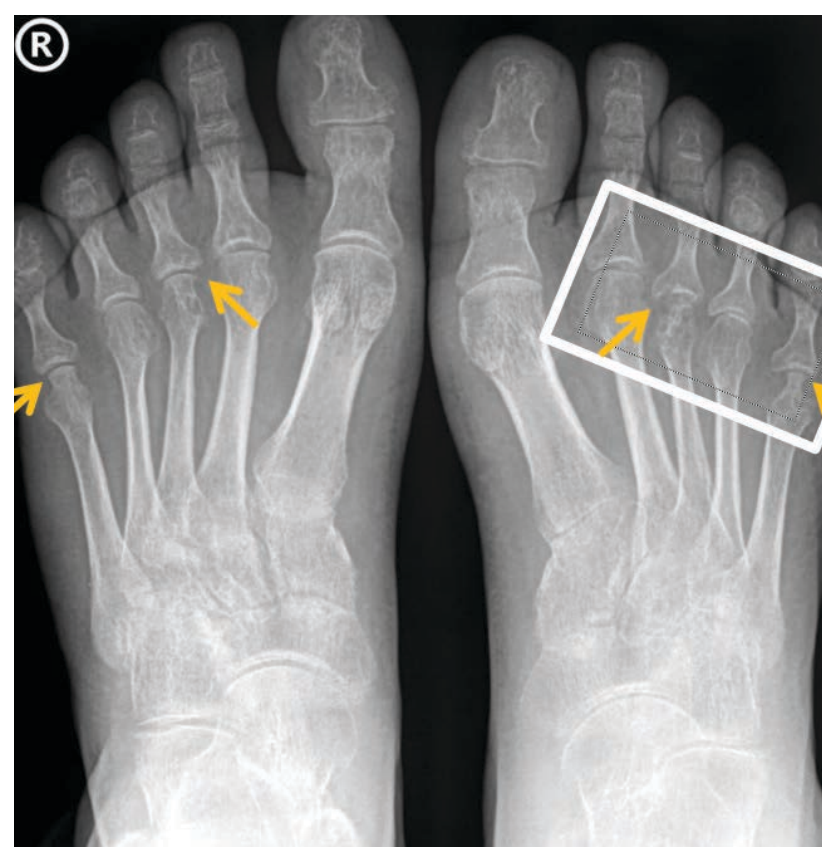

FIGURE 3. Periarticular osteoporosis (white box), bilateral large erosions MTP 3, MTP5 (yellow arrow) in a 33yrs RA female patient, at 3 year of disease onset (delayed diagnosis) (image from the collection of Clinical Center of Rheumatic Diseases "Dr. Ion Stoia")

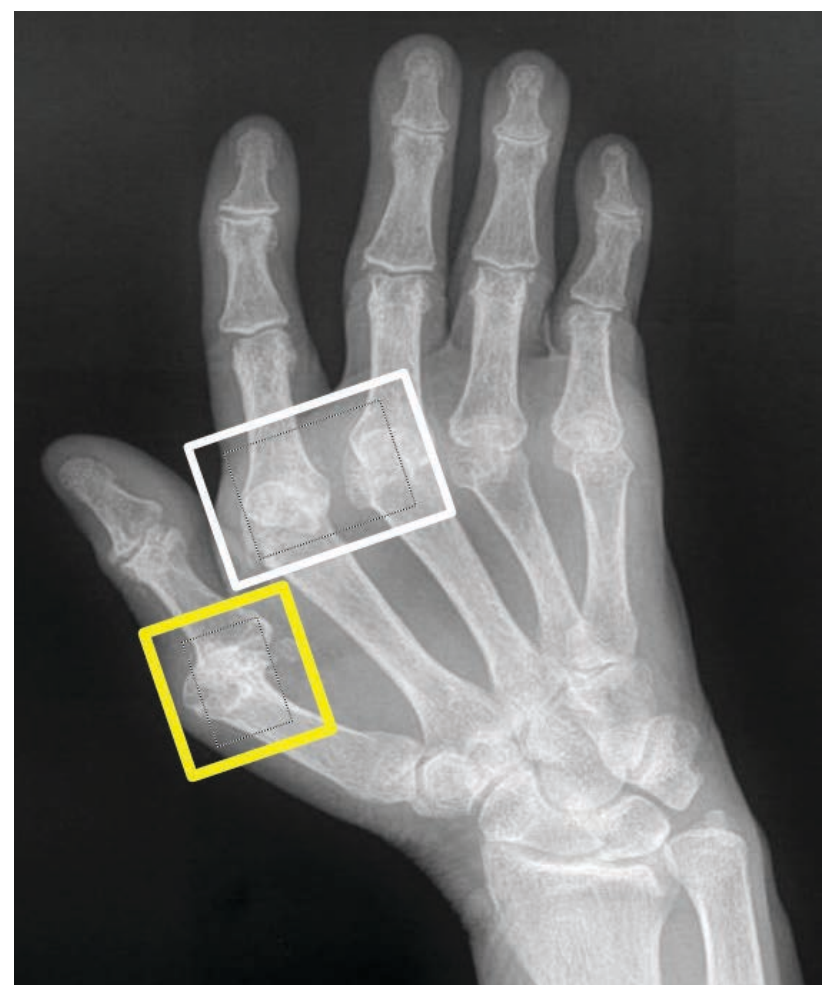

FIGURE 4. Subluxation of the MCP1 (yellow box), anteroposterior luxation of MCP2, MCP3 (white box) in a $67 \mathrm{fe}$ male RA patient at 10 years after disease onset (image from the collection of Clinical Center of Rheumatic Diseases “Dr. Ion Stoia”)

- subchondral geodes are represented by subchondral cysts filled with joint fluid (cystlike lesions) usually small, as a sign of synovitis aggression of the subchondral bone (13);
- specific deformities, subluxations, luxations, as a consequence of the destruction of the joint capsule, ligaments and tendons (Figure 4)

The time course of disease progression in RA is not linear and progression of joint involvement is not uniform. In a single individual, various imaging findings may be present in different joints at any time. Therefore, the most frequently affected joints have to be evaluated (at least the hands, feet and cervical spine) and the symptomatic joints should be included (14).

This observation was further formulated in the EULAR recommendations for the use of imaging of the joints in the clinical management RA, where recommendation number 4 stays that conventional radiograph of the hands and feet should be used as the initial imaging technique to detect damage (15). Radiographic progression in RA has been shown to occur early, and the first erosions are more often found in the feet than in the hands $(16,17,34)$.

One practical problem is how often the radiographic analysis should be performed during RA evolution. Besides the baseline hand and feet $\mathrm{x}$-ray, the EULAR Recommendation number 8 encourage clinicians that the periodic evaluation of joint damage, usually by radiographs of the hands and feet, should be considered (17). Even there were no specific data on the recommended frequency of imaging in the assessment of progressive joint damage, probably because of its variability from case to case, for clinical purposes a time interval of 2 years could be a proper interval to assess progression. During evolution, under specific disease features, each clinician can decide on a shorter or larger time interval.

Radiographs can be used as an outcome measure to assess the severity and progression of RA, to establish the effect of treatment and to provide a permanent record with which the disease can be serially evaluated. An additional advantage of radiographs is that they can be randomised and blinded for standardised scoring (19-24).

\section{RADIOGRAPHIC SCORING METHODS}

The instruments for evaluation of the structural damage on x-ray have been developed since the 50's. The radiographic score includes evaluation of erosions and joint space narrowing. Since the first successfully attempt of describing a radiological scoring system for RA, there many were others developed, as different approach was used: globally evaluation for the entire patient, globally evaluation of certain joints 
or separate evaluation for erosions and joint space narrowing.

Steinbrocker (24) and Kellgren (25) have proposed a global assessment for the entire skeleton, whereas Sharp score (and its variants), Larsen score (and its variants) and Simple Erosion Narrowing Score (SENS) assess individual joints (23).

In 1971, Sharp proposed a scoring method for the hands and wrists, but this version is no longer used (19). A later modification (1985) is now considered the standard for the Sharp method (26). It evaluates 17 areas for erosions and 18 areas for joint space narrowing (JSN) only in hands. The erosion score can vary from one to a maximum of five points for each area. Total erosion scores range from 0 to 170 . For JSN: one point is scored for focal JSN, two points for diffuse narrowing of less than $50 \%$ of the original space and three points if the reduction is more than $50 \%$. Ankylosis is scored as four points. (Sub)luxation is not scored. The score for JSN ranges from 0 to 144 .

In 1986, another modification was devised by Fries et al (with the participation of Sharp) (27).

In 1974, Larsen developed a method based on a set of standard radiologic images (28). It differentiates six stages from 0 (normal) to 5 , reflecting gradual, progressive deterioration, and provides an overall measure of joint damage. This method was modified several times (1977, 1978, 1984, 1985, 1987, and 1995). In the 1977 version, the six stages are as follows: grade $0=$ normal; grade $1=$ slight abnormalities (periarticular soft tissue swelling and periarticular osteoporosis and slight JSN); grade 2 = definite early abnormalities; grade $3=$ medium destructive

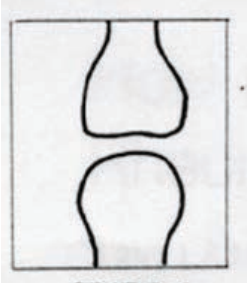

GRADE 0

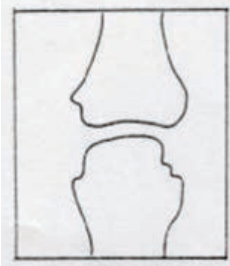

GRADE 3

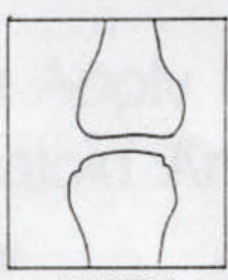

GRADE 1

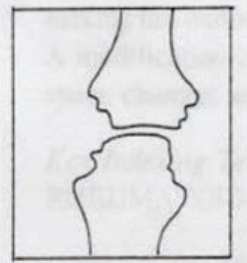

GRADE 4

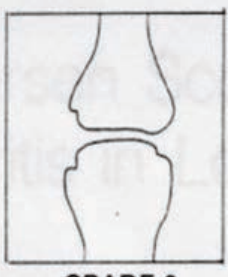

GRADE 2

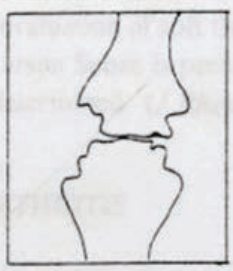

GRADE 5
FIGURE 5. The six stages of Larsen scoring method 1977 version (19) abnormalities; grade $4=$ severe definite abnormalities; and grade $5=$ mutilating abnormalities (Figure 5). The wrist is considered as one unit and the score is multiplied by five. Joints assessed include five distal interphalangeal (DIP), four proximal interphalangeal (PIP), five metacarpo-phalangeal (MCP), the wrist as one unit for each hand and wrist, and 10 metatarso-phalangeal (MTP), two interphalangeal (IP) for the feet. The score ranges from 0 to 250 (19).

In 1983, Genant developed a method of scoring hand and foot radiographs (29); in 1998 he released a modified version, which is in current use (30). The erosion is scored according to an eight point scale with 0.5 increments, where $0=$ normal; $0+=$ questionable or subtle change; $1=$ mild; $1+=$ mild worse; $2=$ moderate; $2+=$ moderate worse $3=$ severe; and $3+=$ severe worse (Figure 6). In each hand, IP of the thumb, PIP, MCP, 1st carpo-metacarpal (CMC), scaphoid, ulna, and radius are included. The score for erosion ranges from 0 to 98 . JSN is scored according to a nine point scale with 0.5 increments, where $0=$ normal; $0+=$ questionable or subtle change; $1=$ mild; $1+=$ mild worse; $2=$ moderate $2+=$ moderate worse; $3=$ severe; $3+=$ severe worse; and $4=$ ankylosis or dislocation. In each hand, IP of the thumb, PIP, MCP, CMC 3 to 5, capitate-scaphoid-lunate, and the radiocarpal joint are included. The score for JSN ranges from 0 to 104. After separately summing the two scores for both hands, each score is normalised to a scale from 0 to 100 (19).

In 1989, van der Heijde modified the method described by Sharp in 1985 (31). Erosion is assessed in 16 joints (five MCP, four PIP, IP of the thumbs, 1st

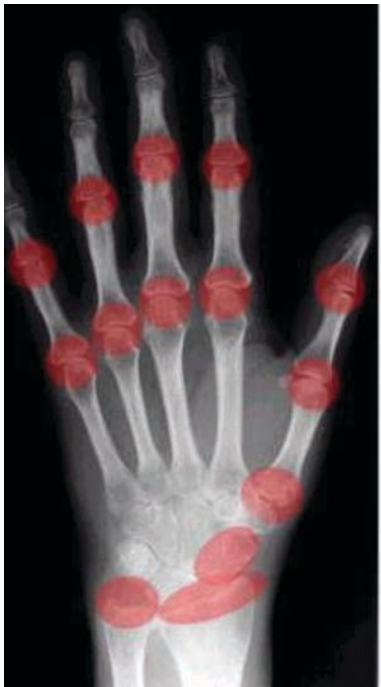

Erosion: 8-point scale with 0.5 increments 14 sites/hand

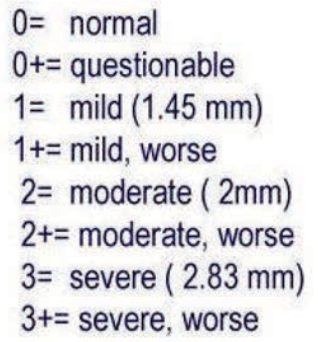

FIGURE 6. Erosion score - Genant modified version 1998 (30) 


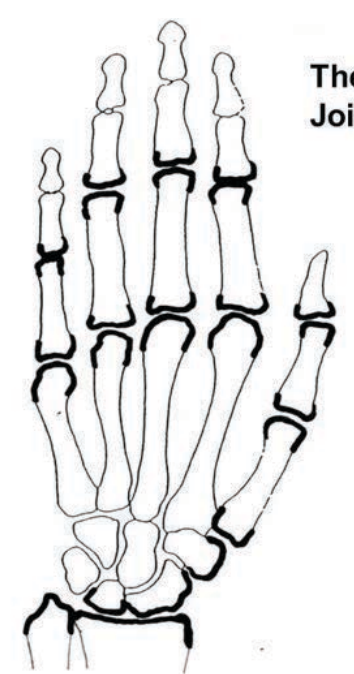

The Sharp/van der Heijde: Joints to be scored for erosions

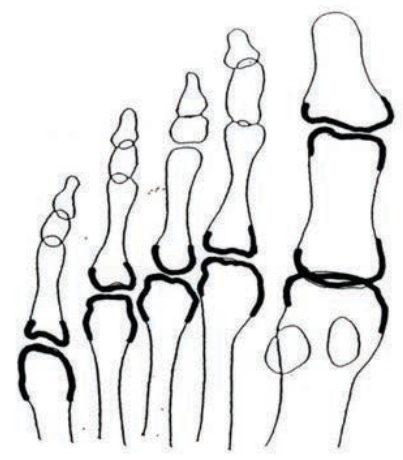

FIGURE 7. Erosion score - Sharp/vand der Heijde method (31)

$\mathrm{CMC}$, radius and ulna bones, trapezium and trapezoid as one unit (multiangular), navicular, lunate) for each hand and wrist, and six joints (five MTP, IP) for each foot (Figure 7). Erosion score ranges from one point for discrete erosion, two points for larger erosion not passing the bone middle line, three points for larger erosion passing the bone middle line, four or five points depending on the amount of surface area affected (complete collapse of the bone is scored as five). The maximum erosion score is $5 /$ joint in hands and 10 / joint in foot. The score for erosion ranges from 0 to 160 in the hands and from 0 to 120 in the feet. JSN is assessed in 15 joints (five MCP, four PIP, CMC 3 to 5, multiangular navicular-lunate, radiocarpal) for each hand and wrist, and six joints (five MTP, IP) for each foot. JSN is combined with a score for (sub)luxation and scored as follows: $0=$ normal; $1=$ focal or doubtful; $2=$ generalised, less than $50 \%$ of the original joint space; $3=$ generalised, more than $50 \%$ of the original joint space or subluxation; 4 = bony ankylosis or complete luxation. The score for JSN ranges from 0 to 120 in the hands and from 0 to 48 in the feet (19).

In 1999, van der Heijde described the SENS method (32). It is a simplified method of scoring radiographs based on the Sharp/van der Heijde score: instead of grading, the number of joints with erosions and with JSN is simply summed. SENS assesses the same joints as the Sharp/van der Heijde method. A joint is scored as affected (1) if it displays any erosion, and as affected (1) for JSN if it scored 1 or more in the original method (at least focal JSN). The score for each joint can therefore range from 0 to 2 . Erosion is assessed in 32 joints in the hands and 12 in the feet, JSN in 30 and 12 joints, respectively. The total SENS score ranges from 0 to 86 (19).
In 1995, Larsen devised a method to evaluate radiographs in long term studies (33). The main differences from the original version are: deletion of scores for the thumbs and 1st MTP; subdivision of the wrist into four quadrants (the joints considered are PIP 2 to 5 and MCP 2 to 5 in each hand, four quadrants in the wrist, and MTP 2 to 5 in each foot); deletion of soft tissue swelling and osteoporosis; distinction between erosions of different sizes. The grading scale ranges from 0 to 5: $0=$ intact bony outlines and normal joint space; 1 = erosion less than $1 \mathrm{~mm}$ in diameter or JSN; $2=$ one or several small erosions (diameter more than $1 \mathrm{~mm}) ; 3=$ marked erosions; $4=$ severe erosions (usually no joint space left and the original bony outlines are only partly preserved); and $5=$ mutilating changes (the original bony outlines have been destroyed). The score ranges from 0 to 160 (19).

With the exception of the Sharp method, all scoring techniques are based on the evaluation of hand and foot joints. Radiographic scoring is used in clinical trials to measure structural changes in RA. When choosing a system scoring the decision is often based on the features included, the joints counted, and the scale used (19). Even if there is no existing scoring method that include all the abnormalities that can be seen on radiographs is RA, erosions and, to a minor grade, JSN are widely accepted as most important.

In clinical trials the readers are blind to the time points order as they assess the radiographs. This can be assessed at a given point in time using absolute scores, or between two time points using values related to progression (19).

\section{SENSITIVITY TO CHANGE}

Sensitivity to change is measured to determine whether a radiographic scoring method can detect a real change over time. Some authors use standardised response mean (SRM), other use the minimal detectable change (MDC) (also called the smallest detectable difference (SDD). SRM is defined as a unitless expression of change and a value above 0.80 is considered to reflect high potential to detect changes. The MDC is also defined as a unitless expression of change, it can range from 0 to 1 , and values above 0.80 are considered to be good $(19,35)$.

\section{CONCLUSIONS}

The main objective of the present review was to present the radiographic evaluation for RA starting from the clinical practice perspective to a broader ap- 
proach, with the most used scoring systems and a clue to interpret figures from clinical trials with respect to structural progression.

From the clinical approach: radiographs of hands and feet should be taken at baseline, as a component of imaging diagnosis; most likely, the first changes are seen in feet (even in asymptomatic patients).

\section{Conflict of interest: none declared}

Financial support: none declared

\section{REFERENCES}

1. Keenan RT, Swearingen CJ, Yazici Y, et al. Erythrocyte sedimentation rate and C-reactive protein levels are poorly correlated with clinical measures of disease activity in rheumatoid arthritis, systemic lupus erythematosus and osteoarthritis patients Clin Exp Rheumatol. 2008;26(5):814-819.

2. Aletaha D, Neogi T, Silman AJ, et al. Rheumatoid arthritis classification criteria: An American College of Rheumatology/ European League Against Rheumatism collaborative initiative. Arthritis Rheum. 2010;62:2569-2581.

3. Nieuwenhuis WP, van Steenbergen HW, Stomp W, et al. The course of bone marrow edema in early undifferentiated arthritis and rheumatoid arthritis: A longitudinal magnetic resonance imaging study at bone level. Arthritis Rheumatol. 2016;68:1080-1088.

4. Kgoebane K, Ally MMTM, Duim-Beytell MC, Suleman FE. The role of imaging in rheumatoid arthritis. S Afr J Rad. 2018;22(1):a1316.

5. Blom M, Creemers M, Kievit W, Lemmens J, van Riel P. Longterm follow-up of the cervical spine with conventional radiographs in patients with rheumatoid arthritis. Scand J Rheumatol. 2013;42(4):281-288

6. Greenspan A, Beltran J, Steinbach LS. Orthopedic imaging: A practical approach. 6 th ed. Philadelphia, PA: Lippincott Williams and Wilkins, 2014

7. Kirwan JR. Links between radiological change, disability, and pathology in rheumatoid arthritis. J Rheumatol. $2001 \mathrm{Apr} ; 28(4): 881-6$.

8. Gossec L, Dougados M, Goupille P, Cantagrel A, Sibilia J, Meyer $\mathrm{O}$, Sany J, Daurès JP, Combe B. Prognostic factors for remission in early rheumatoid arthritis: a multiparameter prospective study. Ann Rheum Dis. 2004 Jun;63(6):675-80.

9. Shiraishi M, Fukuda T, Igarashi T, Tokashiki T, Kayama R, Ojiri H. Differentiating Rheumatoid and Psoriatic Arthritis of the Hand: Multimodality Imaging Characteristics. Radiographics. 2020 SepOct;40(5):1339-1354

10. Colebatch AN, Christopher Edwards CJ, Østergaard M, et al. EULAR recommendations for the use of imaging of the joints in the clinical management of rheumatoid arthritis. Ann Rheum Dis. 2013;72(6):804-814.

11. Brown AK, Quinn MA, Karim Z, et al. Presence of significant synovitis in rheumatoid arthritis patients with diseasemodifying antirheumatic drug-induced clinical remission: evidence from an imaging study may explain structural progression. Arthritis Rheum. 2006;54(12):3761-3773.

12. Böttcher J, Pfeil A. Diagnosis of periarticular osteoporosis in rheumatoid arthritis using digital X-ray radiogrammetry. Arthritis Res Ther. 2008;10(1):103.

13. Jayson MI, Rubenstein D, Dixon AS. Intra-articular pressure and rheumatoid geodes (bone 'cysts'). Ann Rheum Dis. 1970 Sep;29(5):496-502

14. Sommer OJ, Kladosek A, Weiler V, Czembirek H, Boeck M, Stiskal M. Rheumatoid arthritis: a practical guide to state-of-the-art imaging, image interpretation, and clinical implications. Radiographics. 2005 Mar-Apr;25(2):381-98.

15. Colebatch AN, Edwards CJ, Østergaard M, van der Heijde D, Balint PV, D'Agostino MA, Forslind K, Grassi W, Haavardsholm EA, et al. EULAR recommendations for the use of imaging of the joints in the clinical management of rheumatoid arthritis. Ann Rheum Dis. 2013 Jun;72(6):804-14.

16. Andersson ML, Svensson B, Forslind K. Distribution of erosions in hands and feet at the time for the diagnosis of RA and during 8-year follow-up. Clin Rheumatol. 2020 Oct 23.

17. van der Heijde DM, van Leeuwen MA, van Riel PL, van de Putte LB. Radiographic progression on radiographs of hands and feet
Monitoring disease progression with radiographs should be considered at different time intervals, depending on individual factors.

From a clinical trials perspective: the scoring system used could be different, depending on the trial design. The change in score over time in a clinical trial has to use SRM or MDC of above 0.80 .

during the first 3 years of rheumatoid arthritis measured according to Sharp's method (van der Heijde modification). J Rheumatol. 1995 Sep;22(9):1792-6.

18. Larsen A. A radiological method for grading the severity of rheumatoid arthritis. Scand J Rheumatol. 1975;4(4):225-33.

19. Boini S, Guillemin F. Radiographic scoring methods as outcome measures in rheumatoid arthritis: properties and advantages. Ann Rheum Dis. 2001 Sep;60(9):817-27.

20. Sharp JT. Radiologic assessment as an outcome measure in rheumatoid arthritis. Arthritis Rheum. 1989;32:221-9.

21. Brower AC. Use of the radiograph to measure the course of rheumatoid arthritis. The gold standard versus fool's gold. Arthritis Rheum. 1990;33:316-24.

22. Van der Heijde D. Plain X-rays in rheumatoid arthritis: overview of scoring methods, their reliability and applicability. Baillieres Clin Rheum. 1996;10:435-53.

23. Van der Heijde D, Boers M, Lassere M. Methodological issues in radiographic scoring methods in rheumatoid arthritis. J Rheumatol. 1999;26:726-30.

24. Steinbrocker $\mathrm{O}$, Traeger $\mathrm{C}$, Batterman $\mathrm{R}$. Therapeutic criteria in rheumatoid arthritis. JAMA. 1949;140:659-62.

25. Kellgren J, Bier F. Radiological signs of rheumatoid arthritis: a study of observer di Verences in the reading of hand films. Ann Rheum Dis. 1956;15:55-60.

26. Sharp JT, Wolfe F, Mitchell DM, Bloch DA. The progression of erosion and joint space narrowing scores in rheumatoid arthritis during the first twenty-five years of disease. Arthritis Rheum. 1991;34:660-8.

27. Fries JF, Bloch DA, Sharp JT, McShane DJ, Spitz P, Bluhm GB, et al. Assessment of radiologic progression in rheumatoid arthritis. $A$ randomized, controlled trial. Arthritis Rheum. 1986;29:1-9.

28. Larsen A, Dale K, Eek M. Radiographic evaluation of rheumatoid arthritis and related conditions by reference films. Acta Radiol Diagn. 1977;18:481-91.

29. Genant HK. Methods of assessing radiographic change in rheumatoid arthritis. Am J Med. 1983;75:35-47.

30. Genant HK, Jiang Y, Peterfy C, Lu Y, Redei J, Countryman PJ. Assessment of rheumatoid arthritis using a modified scoring method on digitized and original radiographs. Arthritis Rheum. 1998;41:1583-90.

31. van der Heijde DM, van Riel PL, Nuver-Zwart IH, Gribnau FW, vad de Putte LB. Effects of hydroxychloroquine and sulphasalazine on progression of joint damage in rheumatoid arthritis. Lancet. 1989 May 13;1(8646):1036-8.

32. Van der Heijde D, Dankert T, Nieman F, Rau R, Boers M. Reliability and sensitivity to change of a simplification of the Sharp/ van der Heijde radiological assessment in rheumatoid arthritis. Rheumatology (Oxford) 1999;38: 941-7.

33. Larsen A. How to apply Larsen score in evaluating radiographs of rheumatoid arthritis in long-term studies. J Rheumatol. 1995;22:1974-5.

34. Van der Heijde DMFM, Van Leeuven MA, Van Riel PL, Koster AM, Van't Hof MA, Van Rijswijk MH, et al. Biannual radiographic assessments of hands and feet in a three year prospective followup of patients with early rheumatoid arthritis. Arthritis Rheum. 1992;35:26-34.

35. Van der Heijde D, Boonen A, Boers M, Kostense P, van der Linden S. Reading radiographs in chronological order, in pairs or as single films has important implications for the discriminative power of rheumatoid arthritis clinical trials. Rheumatology (Oxford). 1999;38:1213-20. 